Canad. Math. Bull. Vol. 22 (4), 1979

\title{
LOCAL UNIQUE FACTORIZATION IN THE SEMIGROUP OF PATHS IN $\mathbb{R}^{n}$
}

\author{
BY \\ MOHAN S. PUTCHA
}

\begin{abstract}
Let $S$ denote the semigroup of all rectifiable, piecewise continuously differentiable paths in $\mathbb{R}^{n}$ under concatenation. We prove a theorem to the effect that every finite collection of paths is contained in a subsemigroup of $S$ which has the unique factorization property with respect to certain primes and straight lines. We also determine an abstract necessary sufficient condition for a subsemigroup of $S$ to have this unique factorization property.
\end{abstract}

Throughout this paper, $\mathbb{R}, \mathbb{R}^{+}, Z^{+}$will denote the sets of all real numbers, positive reals, positive integers, respectively. $n$ will denote a fixed positive integer and $\mathbb{R}^{n}$ the Euclidean $n$-space. Let $M$ denote the set of all rectifiable, piecewise continuously differentiable functions $f$ from $[0,1]$ into $\mathbb{R}^{n}$ such that $f(0)=0$ and $f$ is not constant on any subinterval of $[0,1]$. If $f, g \in \mathcal{M}$, then let $f g \in \mathcal{M}$ be defined by

$$
f g(x)= \begin{cases}f(2 x) & 0 \leq x \leq \frac{1}{2} \\ f(1)+g(2 x-1), & \frac{1}{2} \leq x \leq 1\end{cases}
$$

If $f, g \in \mathcal{M}$, then define $f \equiv g$ if $g=f \circ \phi$ for some strictly increasing, continuous self-map $\phi$ of $[0,1]$ with $\phi(0)=0, \phi(1)=1$. Then $S=M / \equiv$ is a cancellative semigroup (see $[2,3]$ where $\mathscr{D}_{1}^{*}$ was used to denote this semigroup). Let $\mathscr{L}$ denote the set of all lines in $S$. If $u \in S$, then $l(u)$ denotes the length of $u$. If $a \in \mathscr{L}, \alpha \in \mathbb{R}^{+}$, then let $a^{\alpha}$ denote the line parallel to $a$ having length $\alpha l(a)$. If $a, b \in \mathscr{L}$, then we will write $a \sim b$ if $a$ is parallel to be (i.e., $b=a^{\alpha}$ for some $\alpha \in \mathbb{R}^{+}$). If $A \subseteq S$, then let $\langle A\rangle$ denote the semigroup generated by $A$. If $\Gamma$ is a set, then let $\mathscr{F}=\mathscr{F}(\Gamma)$ denote the free semigroup on $\Gamma$. If $\Lambda \subseteq \Gamma$, then let $\mathscr{F}_{\mathbb{R}}(\Gamma \mid \Lambda)$ denote the semigroup of words in alphabet $\Gamma$ such that for $A \in \Gamma \backslash \Lambda$ the exponents of $A$ in the word are allowed to be positve real numbers. So $\mathscr{F}_{\mathbb{R}}(\Gamma \mid \Gamma)=\mathscr{F}(\Gamma)$ and $\mathscr{F}_{\mathbb{R}}=\mathscr{F}_{\mathbb{R}}(\Gamma)=\mathscr{F}_{\mathbb{R}}(\Gamma \mid \varnothing)$ is the free product of $|\Gamma|$ copies of positive reals under addition (see [3]). Let $S^{1}$ be the semigroup $S \cup\{1\}, 1 \notin S$ such that 1 is the identity element of $S^{1}$. If $X \subseteq S$, then $X^{1}=X \cup\{1\}$.

If $X \subseteq S$, then the power closure of $X, \quad \bar{X}=\left\{u^{i} \mid u \in X\right.$, $\left.i \in \mathbb{Z}^{+}\right\} \cup\left\{u^{\alpha} \mid u \in X \cap \mathscr{L}, \alpha \in \mathbb{R}^{+}\right\}$. Let $a, b \in S, X, Y \subseteq S$. Then $a<_{X, Y} b$ is $b=$ $x a y$ for some $x, y \in S^{1}$ such that $(x, y) \in\left(X^{1} \times X^{1}\right) \backslash\left(Y^{1} \times Y^{1}\right)$. Let $T$ be a subsemigroup of $S$. Then $T$ satisfies the descending chain condition if there is

Received by the editors April 3, 1978 and, in revised form, October 18, 1978. 
no sequence in $T$ of the following type

$$
\cdots \underset{T, \mathscr{L}}{<} a_{3}<{ }_{T, \mathscr{L}} a_{2} \underset{T, \mathscr{L}}{<} a_{1}
$$

$T$ is a weakly unitary subsemigroup of $S$ if for all $a \in S$, the conditions $a T \cap T \neq \varnothing$ and $T a \cap T \neq \varnothing$, together imply $a \in T$. This condition, due to Schützenberger, comes up naturally in the study of free semigroups [1; p. 119]. $T$ is power closed if $\bar{T}=T . T$ is free-like if $T$ is weakly unitary, is power closed and satisfies the descending chain condition.

REMARK. Intersection of free-like subsemigroups of $S$ is again free-like.

THEOREM 1. Let $T$ be a free-like subsemigroup of $S$. Then $T \cong \mathscr{F}_{\mathbb{R}}(\Gamma \mid \Lambda)$ for some $\Gamma, \Lambda$.

Proof. Let $\mathscr{L}_{t}=T \cap \mathscr{L}$. Then $T \backslash \mathscr{L}_{t}$ is a subsemigroup of $T$. Let $D=$ $\left\{a \mid a \in T \backslash \mathscr{L}_{t}, a \neq b c\right.$ for any $\left.b, c \in T \backslash \mathscr{L}_{t}\right\}$. We first show that $T \backslash \mathscr{L}_{t}=\langle D\rangle$. For suppose $a \in T \backslash \mathscr{L}_{t}, a \notin\langle D\rangle$. Then $a=b c$ for some $b, c \in T \backslash \mathscr{L}_{t}$. So $b<_{T, \mathscr{L}} a$, $c<_{T, \mathscr{L}} a$. Either $b \notin\langle D\rangle$ or $c \notin\langle D\rangle$. Thus there exists $a_{1} \in T \backslash \mathscr{L}_{t}$ such that $a_{1} \notin\langle D\rangle, a_{1}<_{T, \mathscr{L}} a$. Continuing, we find a sequence $\left\{a_{i}\right\}_{i \in \mathbb{Z}^{+}}$in $T \backslash\left(\mathscr{L}_{t} \cup\langle D\rangle\right)$ such that

$$
\cdots \underset{T, \mathscr{L}}{<} a_{2} \underset{T, \mathscr{L}}{<} a_{1} \underset{T, \mathscr{L}}{<} a .
$$

This violates the descending chain condition of $T$. So $T \backslash \mathscr{L}_{t}=\langle D\rangle$. Let $P_{1}=$ $\left\{a \mid a \in D, a \notin b T\right.$ for any $\left.b \in \mathscr{L}_{t}\right\}, P_{2}=\left\{a \mid a \in D, a \notin T b\right.$ for any $\left.b \in \mathscr{L}_{t}\right\}, P=$ $P_{1} \cap P_{2}$. We claim that $D \subseteq\left\langle\mathscr{L}_{t}\right\rangle^{1} P_{1}^{1}$. For suppose $a \in D, a \notin\left\langle\mathscr{L}_{t}\right\rangle^{1} P_{1}^{1}$. Then there exists $b_{1} \in \mathscr{L}_{t}$ such that $a=b_{1} a_{1}, a_{1} \in T \backslash \mathscr{L}_{t}, a_{1} \notin b_{1}^{\alpha} T$ for any $\alpha \in \mathbb{R}^{+}$. Then clearly $a_{1} \in D$ and so $a_{1} \notin\left\langle\mathscr{L}_{t}\right\rangle^{1} P_{1}^{1}$. Continuing we find a sequence $\left\{b_{i}\right\}_{i \in \mathbb{Z}^{+}}$in $\mathscr{L}_{t}$, $\left\{a_{i}\right\}_{i \in \mathbb{Z}^{+}}$in $D$ such that $b_{i} x b_{i+1}$ for any $i$ and for any $i \in Z^{+}, a_{i}=b_{i+1} a_{i+1}$. So $a_{i}=b_{i+1} b_{i+2} a_{i+2}$ and $a_{i+2}<_{T, \mathscr{L}} a_{i}$. So

$$
\cdots \underset{T, \mathscr{L}}{<} a_{6} \underset{T, \mathscr{L}}{<} a_{4} \underset{T, \mathscr{L}}{<} a_{2} \underset{T, \mathscr{L}}{<} a .
$$

This violates the descending chain condition of $T$. Hence, $D \subseteq\left\langle\mathscr{L}_{t}\right\rangle^{1} P_{1}^{1}$. Similarly $D \subseteq P_{2}^{1}\left\langle\mathscr{L}_{t}\right\rangle^{1}$. Let $a \in D$. Then $a=b c$ for some $b \in\left\langle\mathscr{L}_{t}\right\rangle^{1}, c \in P_{1}^{1}$. Now $c=d h$ for some $d \in P_{2}^{1}, h \in\left\langle\mathscr{L}_{t}\right\rangle^{1}$. Since $c \in P_{1}^{1}, d \in P_{1}^{1} \cap P_{2}^{1}=P^{1}$. So $a=b d h, b$, $h \in\left\langle\mathscr{L}_{t}\right\rangle^{1}, d \in P^{1}$. Thus $D \subseteq\left\langle\mathscr{L}_{t}\right\rangle^{1} P^{1}\left\langle\mathscr{L}_{t}\right\rangle^{1}$. Since $T \backslash \mathscr{L}_{t}=\langle D\rangle$ we see that $T=$ $\left\langle P \cup \mathscr{L}_{t}\right\rangle$. Let $\mathscr{L}_{u}=\left\{a \mid a \in \mathscr{L}_{t}, l(a)=1\right\}$. If $a, b \in \mathscr{L}_{u}$, then $a \sim b$ implies $a=b$. Also $\overline{\mathscr{L}}_{u}=\mathscr{L}_{t}$. So clearly $\left\langle\mathscr{L}_{t}\right\rangle \cong \mathscr{F}_{\mathbb{R}}\left(\mathscr{L}_{u}\right)$. Also it is clear that $T=\left\langle P \cup \overline{\mathscr{L}}_{u}\right\rangle$. Let $\Gamma=P \cup \mathscr{L}_{u}, \Lambda=P$. We claim that $T \cong \mathscr{F}_{\mathbb{R}}(\Gamma \mid \Lambda)$. To see this, let $a \in T, a=$ $a_{1} \cdots a_{m}=b_{1} \cdots b_{p}$ where $a_{1}, \ldots, a_{m}, b_{1}, \ldots, b_{p} \in P \cup \mathscr{L}_{t}$ such that if $a_{i}$, $a_{i+1} \in \mathscr{L}_{t}$, then $a_{i} x a_{i+1}$ and if $b_{j}, b_{j+1} \in \mathscr{L}_{t}$, then $b_{i} x b_{j+1}$. We must show that $m=p$ and $a_{i}=b_{i}$ for all $i$. Let $u=a_{2} \cdots a_{m}, v=b_{2} \cdots b_{p}$. Then $a_{1} u=b_{1} v$. First suppose $a_{1} \in \mathscr{L}_{t}$. We claim that $b_{1} \in \mathscr{L}_{t}$. For suppose $b_{1} \in P$. Since $b_{1} \notin \mathscr{L}_{t}$, 
$l\left(b_{1}\right)>l\left(a_{1}\right)$. So $b_{1}=a_{1} c$ for some $c \in S$. Then $a_{1}, v, a_{1} c, c v=u \in T$. Since $T$ is weakly unitary, $c \in T$. This contradicts the fact that $b_{1} \in P$. So $b_{1} \in \mathscr{L}_{t}$. Then clearly $a_{1} \sim b_{1}$. We claim that $a_{1}=b_{1}$. Otherwise by symmetry assume $l\left(a_{1}\right)<$ $l\left(b_{1}\right)$. Then $b_{1}=a_{1} c$ for some $c \in \mathscr{L}$. Since $c \sim a_{1}, T$ is power closed, $c \in \mathscr{L}_{t}$. Also,

$$
a_{2} \cdots a_{m}=c v \text {. }
$$

As above, $a_{2} \in \mathscr{L}_{t}, a_{2} \sim c \sim a_{1}$, a contradiction. So $a_{1}=b_{1}$. Next assume $a_{1}$, $b_{1} \in P$. Suppose $l\left(a_{1}\right)<l\left(b_{1}\right)$. Then $b_{1}=a_{1} c$ for some $c \in S$. So $a_{1} c, a_{1}, c v=u$, $v \in T$ and so $c \in T$. If $c \in \mathscr{L}_{t}$, we get a contradiction to the fact that $b_{1} \in P$. Otherwise we get a contradiction to the fact that $b_{1} \in D$. Thus $a_{1}=b_{1}$ in all cases. We are now done by induction.

REMARK. Let $T$ be a free-like subsemigroup of $S, T \cong \mathscr{F}_{\mathbb{R}}(\Gamma \mid \Lambda)$. If the elements of $\Lambda$ are thought of as primes, then $T$ has the unique factorization property with respect to primes and lines.

Corollary 2. Let $T$ be a subsemigroup of $S$ such that $T \cap \mathscr{L}=\varnothing$. Then $T$ is free if and only if $T$ is free-like.

REMARK. The converse of Theorem 1 is false for the following reason. Let $K$ be a proper subsemigroup of $\left(\mathbb{R}^{+},+\right)$such that $K \cong\left(\mathbb{R}^{+},+\right)$. Let $u \in \mathscr{L}$ and set $T=\left\{u^{\alpha} \mid \alpha \in \mathbb{R}^{+}\right\}$. Then clearly $T \cong\left(\mathbb{R}^{+},+\right)$but $T$ is not free-like.

THEOREM 3. Let $T$ be a power-closed subsemigroup of $S$. If $T \cong \mathscr{F}_{\mathbb{R}}(\Gamma \mid \Lambda)$ for some $\Gamma, \Lambda$, then $T$ is free-like.

Proof. Let $\mathscr{L}_{t}=T \cap \mathscr{L}$. Let $\phi: T \rightarrow \mathscr{F}_{\mathbb{R}}(\Gamma \mid \Lambda)$ be the given isomorphism. Let $a \in S, \quad b, c \in T$ such that $a b, c a \in T$. Then $(c a) b=c(a b)$. So $\phi(c a) \phi(b)=$ $\phi(c) \phi(a b)$. There exists $u \in \mathscr{F}_{\mathbb{R}}(\Gamma \mid \Lambda)$ such that either $\phi(c a)=\phi(c) u$ or $\phi(c)=$ $\phi(c a) u$. Let $a_{1}=\phi^{-1}(u) \in T$. Then $c a a_{1}=c$ or $c a=c a_{1}$. First case being ruled out, $a=a_{1} \in T$. So $T$ is weakly unitary in $S$. Let $\mathscr{K}=\left\{A^{\alpha} \mid A \in \Gamma \backslash \Lambda, \alpha \in \mathbb{R}^{+}\right\}$. If $a, b \in \mathscr{F}_{\mathbb{R}}(\Gamma \mid \Lambda)$, then define $a<b$ if $b=x a y$ for some $x, y \in \mathscr{F}_{\mathbb{R}}(\Gamma \mid \Lambda)^{\mathbf{1}}$ such that $(x, y) \notin \mathscr{K}^{1} \times \mathscr{K}^{1}$. Clearly $\left(\mathscr{F}_{\mathbb{R}}(\Gamma \mid \Lambda),<\right)$ satisfies the descending chain condition. Thus to show that $T$ satisfies the descending chain condition, we must show that $\phi\left(\mathscr{L}_{1}\right)=\mathscr{K}$. If $a \in \mathscr{L}_{t}$, then for each $i \in Z^{+}$, there exists $a_{i} \in T$, such that $a_{i}^{i}=a$. Moreover by the author [2], the elements of $\mathscr{L}_{t}$ are characterized by this property. The same holds true for $\mathscr{K}$ in $\mathscr{F}_{\mathbb{R}}(\Gamma \mid \Lambda)$. This proves that $\phi\left(\mathscr{L}_{1}\right)=\mathscr{K}$, completing the proof.

THEOREM 4. Every finite subset of $S$ is contained in a free-like subsemigroup of $S$.

Proof. Let $a_{1}, \ldots, a_{m} \in S$. Let $k$ be the smallest non-negative integer such that there exist $b_{1}, \ldots, b_{k} \in S$ such that $a_{1}, \ldots, a_{m} \in\left\langle b_{1}, \ldots, b_{k}, \mathscr{L}\right\rangle(k=0$ 
means $\left.a_{1}, \ldots, a_{m} \in\langle\mathscr{L}\rangle\right)$. Let $\mathscr{L}_{u}=\{a \mid a \in \mathscr{L}, l(a)=1\}, T=\left\langle b_{1}, \ldots, b_{k}, \overline{\mathscr{L}}_{u}\right\rangle=$ $\left\langle b_{1}, \ldots, b_{k}, \mathscr{L}\right\rangle$. We will show that $T$ is free-like. Let $\Gamma=\left\{b_{1}, \ldots, b_{k}\right\} \cup \mathscr{L}_{u}$, $\Lambda=\left\{b_{1}, \ldots, b_{k}\right\}$. We claim that $T \cong \mathscr{F}_{\mathbb{P}}(\Gamma \mid \Lambda)$. Suppose not. Then it is easily seen that there exist $A_{1}, \ldots, A_{r}, B_{1}, \ldots, B_{s} \in \Lambda \cup \mathscr{L}$ such that

$$
A_{1} \cdots A_{r}=B_{1} \cdots B_{s}
$$

and either $A_{1}$ or $B_{1} \in \Lambda$ and so that if both $A_{1}, B_{1} \in \Lambda$, then $A_{1} \neq B_{1}$. Let $\left\{A_{1}, \ldots, A_{r}, B_{1}, \ldots, B_{s}\right\} \cap \mathscr{L}=\left\{c_{1}, \ldots, c_{t}\right\}$. Introduce variables $x_{1}, \ldots, x_{k}$, $y_{1}, \ldots, y_{t}$, and words $w_{1}=w_{1}\left(x_{1}, \ldots, x_{k}, y_{1}, \ldots, y_{t}\right), \quad w_{2}=w_{2}\left(x_{1}, \ldots, x_{k}\right.$, $\left.y_{1}, \ldots, y_{t}\right)$ such that $A_{1} \cdots A_{r}$ is formally equal to $w_{1}\left(b_{1}, \ldots, b_{k}, c_{1}, \ldots, c_{t}\right)$ and $B_{1} \cdots B_{s}$ is formally equal to $w_{2}\left(b_{1}, \ldots, b_{k}, c_{1}, \ldots, c_{t}\right)$. We can express $\{1, \ldots, t\}$ as a disjoint union of $T_{1}, \ldots, T_{p}$ such that for $\alpha, \beta \in\{1, \ldots, t\} c_{\alpha} \sim c_{\beta}$ if and only if $\alpha, \beta$ lie in same $T_{j}$. For $j=1, \ldots, p$, let $M_{j}=\left\{\left(y_{j}, l\left(c_{j}\right) \mid j \in T_{j}\right\}\right.$. In the notation of [3], consider the constrained word equation $\mathscr{A}=$ $\left\{w_{1}, w_{2} ; M_{1}, \ldots, M_{p}\right\}$ in free variables $x_{1}, \ldots, x_{k}$ and constrained variables $y_{1}, \ldots, y_{t}$. Then $\mu=\left(b_{1}, \ldots, b_{k}, c_{1}, \ldots, c_{t}\right)$ is a solution of $\mathscr{A}$. By [3; Theorem 5.2], $\mu$ follows from a solution $\nu$ of $\mathscr{A}$ in some $\mathscr{F}_{\mathbb{R}}\left(\Gamma^{\prime} \mid \Lambda^{\prime}\right)$. Moreover a close examination of the proof of [3; Lemma 3.13], shows that in fact we can choose $\Lambda^{\prime}$ such that $\left|\Lambda^{\prime}\right|<k$ (this is because of the non-triviality of (1)). There exists $\phi: \Gamma^{\prime} \rightarrow S, \phi\left(\Gamma^{\prime} \backslash \Lambda^{\prime}\right) \subseteq \mathscr{L}$, such that the natural extension $\hat{\phi}: \mathscr{F}_{\mathbb{R}}\left(\Gamma^{\prime} \mid \Lambda^{\prime}\right) \rightarrow S$ has the property that if $\nu=\left(u_{1}, \ldots, u_{k}, v_{1}, \ldots, v_{t}\right)$, then $\hat{\phi}\left(u_{i}\right)=b_{i}, \hat{\phi}\left(v_{j}\right)=c_{j}$. Let $\phi\left(\Lambda^{\prime}\right)=\left\{d_{1}, \ldots, d_{\theta}\right\}$. So $\theta<k$. Also $b_{1}, \ldots, b_{k} \in \hat{\phi}\left(\mathscr{F}_{\mathbb{R}}\left(\Gamma^{\prime} \mid \Lambda^{\prime}\right)\right)=$ $\left\langle\phi\left(\Lambda^{\prime}\right) \cup \overline{\left.\phi\left(\Gamma^{\prime} \backslash \Lambda^{\prime}\right)\right\rangle}\right.$. So $b_{1}, \ldots, b_{k} \in\left\langle d_{1}, \ldots, d_{\theta}, \mathscr{L}\right\rangle$. Hence $a_{1}, \ldots, a_{m} \in$ $\left\langle d_{1}, \ldots, d_{\theta}, \mathscr{L}\right\rangle$ contradicting the minimality of $k$. This contradiction shows that $T \cong \mathscr{F}_{\mathbb{R}}(\Gamma \mid \Lambda)$. We are done by Theorem 3 .

Remark. Let $a_{1}, \ldots, a_{m} \in S$. Then by the above theorem and the remark preceding Theorem 1 , there is a unique minimal free-like subsemigroup of $S$ containing $a_{1}, \ldots, a_{m}$.

$\mathscr{F}_{\mathbb{R}}(\Gamma)$ is clearly embeddable in a group (in fact in the free product of $|\Gamma|$ copies of reals under addition). So by [1, Theorem 12.6], we have,

THEOREM 5. S is embeddable in a group.

Conjecture. Let $T$ be a subsemigroup of $S$. Then $T$ can be embedded in $\mathscr{F}_{\mathbb{R}}(\Gamma)$ for some $\Gamma$ if and only if $T$ satisfies the descending chain condition.

ExAmple. We give an example of a subsemigroup $T$ of $S$ such that $T$ is embeddable in a free semigroup but $T$ is not contained in a free-like subsemigroup of $S$. We can choose sequences $a_{1}, a_{2}, \ldots, b_{1}, b_{2}, \ldots, c_{1}, c_{2}, \ldots$, in $S \backslash \mathscr{L}$ such that the following properties are true: $(1) a_{i+1} b_{i}=a_{i}, i=1,2, \ldots$, (2) $l\left(c_{i}\right) \geq 3 l\left(a_{i}\right), i=1,2, \ldots$, (3) no segment of $b_{i}$ is a segment of $b_{j}$ for $i \neq j$, (4) no segment of $c_{i}$ is a segment of $c_{j}$ for $i \neq j$, and (5) $b_{i}$ is not an initial segment of $a_{j}$ for any $i, j$. Let $T$ be the subsemigroup of $S$ generated by $c_{i}, c_{i} a_{i}, a_{i} c_{i}, c_{i+1} b_{i}$, 
$b_{i} c_{i+1}, i=1,2, \ldots$ We claim that $T$ is not contained in any free-like subsemigroup of $S$. For suppose $T \subseteq R \subseteq S$ and $R$ is free-like. Then clearly $a_{i}, b_{i} \in T$ for all $i$. So

$$
\cdots \underset{\mathrm{T}, \mathscr{L}}{<} a_{3} \underset{\mathrm{T}, \mathscr{L}}{<} a_{2} \underset{\mathrm{T}, \mathscr{L}}{<} a_{1}
$$

a contradiction. On the other hand $T$ can be embedded in a free semigroup. To see this, let $\mathscr{F}$ be the free semigroup on the letters $A_{1}, A_{2}, \ldots, B_{1}, B_{2}, \ldots$, $C_{1}, C_{2}, \ldots$ Let $K$ be the subsemigroup of $\mathscr{F}$ generated by $C_{i}, C_{i} A_{i}, A_{i} C_{\mathrm{I}}$, $C_{i+1} B_{i}, B_{i} C_{i+1}, i=1,2, \ldots$ Then it can be shown that $T \cong K$ with $c_{i}, c_{i} a_{i}, a_{i} c_{i}$, $c_{i+1} b_{i}, b_{i} c_{i+1}$, corresponding to $C_{i}, C_{i} A_{i}, A_{i} C_{i}, C_{i+1} B_{i}, B_{i} C_{i+1}$, respectively.

\section{REFERENCES}

1. A. H. Clifford and G. B. Preston, The algebraic theory of semigroups, Vol. 2 Amer. Math. Soc., Providence, R.I., 1967.

2. M. S. Putcha, Word equations of paths, Journal of Algebra, (accepted).

3. - Word equations in some geometric semigroups, Pacific Journal of Mathematics, 75 (1978) 243-266.

Department of Mathematics

North Carolina State University

Raleigh, North Carolina 27650

U.S.A. 